\title{
The Curricular Practical Training Rotation Problem Formulation and the Assessment of Rotation Strategies
}

\author{
Ahmet Bahadır Şimşek ${ }^{1, *}$ \\ ${ }^{1}$ Faculty of Department of Health Sciences, University of Gümüşhane, Gümüşhane, Turkey \\ E-mail:〈abahadirsimsek@gumushane.edu.tr〉
}

\begin{abstract}
This study addresses the curricular practical training rotation problem, which is a type of staff assignment problem. Many educational institutions require theoretical knowledge to be complemented by practical training. Although the details of the implementation differ from institution to institution, it is necessary to prepare a rotation plan that determines how long the trainees will practice in which unit in which training period. Because of the complexity of the problem and humanistic reasons, the manual rotation plan can not reach the optimal level that satisfies all stakeholders and takes time. This study defines a general Curricular Practical Training Rotation Planning Problem specific to the curriculum-based trainee assignment process carried out in a university department and proposes an integer mathematical model for its solution. It is one of the important contributions of this study. It also provides a methodological approach to identify the most appropriate rotation strategy that will satisfy stakeholders. The methodological approach followed is a structure that can be adapted to different perspectives. The study has the potential to guide practitioners and researchers in the field and to lead a rich literature that will be formed with different side constraints and purposes to the problem.
\end{abstract}

Keywords: Curricular Practical Training Rotation Planning, Rotation Strategy, Integer Programming, Simple Weighted Method

Received: May 24, 2021; accepted: June 29, 2021; available online: December 23, 2021

DOI: $10.17535 /$ crorr.2021.0010

\section{Introduction}

Practical training is an important stage of education. The aim is for students to consolidate and internalize their theoretical knowledge by practicing. Many education systems offer students two practical training options: Optional Practical Training (OPT) and Curricular Practical Training $(\mathrm{CPT})$. OPT is a customizable and optional program, but CPT is part of the curriculum required for graduation. The compulsory nature of the CPT combines many benefits and challenges. It provides students a variety of side benefits such as specialization, networking, and career planning, as well as enabling them to complete the program. It is known that experiences and references are important, especially in job applications after education. At this point, CPT offers an important opportunity for students to gain an advantage over their rivals. During education, students have the chance to practice many times in institutions that have signed protocols with educational institutions. In this way, they acquire gains that they could not have outside of the CPT. On the other hand, the student may be faced with the following difficulties: the units to receive training may not be of interest, may have personal problems with employees, or the training time may be shorter than desired. With good planning, the benefits can be increased by reducing the difficulties.

${ }^{*}$ Corresponding author. 
CPT takes place in rotation so that students gain experience in units specializing in different branches of the relevant field. The rotation plan shows the unit and period in which each student will train and is prepared by an educator. While creating the plan, the number and the preference of students, the trainee capacity of the units, the curriculum, and other side constraints are taken into account as a whole. It is difficult to complete this task successfully by satisfying all stakeholders.

The CPT has three stakeholders: the students, the institution, and the educator. The educator represents the educational institution in which the student is enrolled, while the institution indicates the external partner in which the student is trained. We call students who will attend practical training as a trainee. To explain the complexity of designing a rotation plan that satisfies stakeholders, the conflicting interests of the stakeholders can be explained as follows. The educator would like trainees to be able to practice in all possible units to be competent in their field. However, due to the limited capacity of the units and the limited number of training periods, a trainee can not find the chance to practice in all units. Therefore, the educator chooses trainees to practice in each unit during each practical training period, which means that every trainee has to graduate by practicing in a limited number of units. For the institution, the case is the opposite. The institution bears the costs of introducing, teaching the operation of the unit for each naive trainee and involve in the process. To ensure continuity and reduce costs, the institution wants the same trainee to be assigned to the same unit at each period. In addition, the institution restricts the number of trainees that each unit will accept, as too many trainees may prevent the unit from carrying out its responsibilities. The interests of the institution and the educator are in complete conflict. On the other hand, trainees may be non-selective between units or specifically willing (reluctant) only for a single or a few units. In the worst-case scenario, trainees may have to practice in undesired units.

The educator seeks to design a high-quality rotation plan by considering the above-mentioned conflicts. We define this problem as Curricular Practical Training Rotation Planning Problem (CPTRPP). CPTRPP is a specific variant of multi-period staff assignment problems [1]. The following problems are related to multi-period staff assignment problems: scheduling vehicle drivers [3, 2], audit staff planning $[4,5]$, technician routing and scheduling problem $[7,6]$, medical residents rotation $[9,8]$. CPTRPP is similar to the medical residents' rotation problem (MRRP) in terms of its basic dynamics [1]. The difference in MRRP is that trainees need to fully experience delineated units to have different skill certificates. For the similarity, we share the resident scheduling literature. As far as we know, Franz and Miller [8] first studied MRRP. One of the most prominent sources of motivation in the literature is that manually prepared rotation plans are far from the optimum level. Most studies refer to the automation of the rotation plan as an important outcome of their research [10, 12, 11]. Automation allows comparing the quality of different rotation strategies that cover several settings that reflect different perspectives, and determination of the most suitable strategy [13, 14]. Considering the quality of education is a marked feature $[15,12]$. Flexible shift planning and experience differences of the trainees are the other features considered $[16,17,11]$. Since the success of practical training is directly related to the willingness of the trainee, many studies have tried to satisfy the preferences of the trainees as much as possible $[8,19,18,11]$.

Curricular Practical Training is subject to many restrictions affecting the decision environment such as legal regulations and managerial rules. Rotation strategies created by different combinations of rules represent trade-offs between stakeholders. For example, a strategy that allows the student to be assigned to the same unit for all training periods while pleasing the institution, does not satisfy the educator, or vice versa. Many factors such as the number of students of the educational institution and the capacity of the institutions with which the protocol has signed affect the rotation strategy. The educator should determine the strategy best suited to the circumstances. Creating a rotation plan, which is error-prone and time-consuming when done manually, can become impossible when different strategies need to be tried. This 
is why the automation of the rotation plan is important because it allows both the optimal decision to be made and the testing of different strategies easily.

This study handles the CPTRPP adapted to the specific needs expressed by the Faculty of Health Sciences of the University of Gümüshane in Turkey, Department of Health Management. We offer an automated approach with integer programming to increase the efficiency of the rotation planning process and for optimal planning that will satisfy stakeholders. Apart from automating manual scheduling, the study involves comparing and analyze various rotation strategies. The rotation strategies are designed by combining different sets of rules and various training periods. The comparison is based on three criteria: the level of satisfaction of preferences, the number of training units, and the number of training days in the trained units. We identify the most appropriate strategy and its alternatives with a simple weighting approach for decision-makers with different perspectives.

Contributions of the study can be listed as follows; i) it provides a general mathematical formulation to the curricular practical training rotation problem faced by educational institutions, ii) it investigates different rotation strategies and suggests a methodology for determining the best, iii) it constitutes a source of inspiration for practitioners and researchers in the field and sets an example for similar problems.

The remainder of this paper is structured as follows. Section 2 describes the studied problem, the rotation strategies and also sets out the mathematical model. Section 3 analyses the comparison of rotation strategies. Section 4 contains the conclusions.

\section{Case Detail}

The decision environment in our study can be explained as follows. In the Turkish education system, the CPT is carried out under the curriculum in the framework of a practical training protocol between educational institutions and private or public institutions. The range of training units is limited by the scope of the protocol. Depending on the curriculum, the student practices training in one or more training periods in the same or different units. In general, the main objective is to ensure that trainees are trained in as many different units as possible. An educator in charge of the educational institution prepares a rotation plan showing which unit of the institution in which period the student will practice considering the trainee capacities of the units. The number and duration of practical training sessions are variable, but 3-period settings are commonly used: summer (21 days), fall (14 days), and spring (14 days) semesters. The number of trainees accepted by the units varies depending on their workload.

Our CPTRPP is customized according to the needs expressed by the Health Management Department of the Faculty of Health Sciences of Gumushane University. The university has a training protocol with 4 public institutions, eg I1-Public Hospitals Administration, I2-City Health Administrative, etc. A total of 16 different units in which the trainee can practice are available in these institutions. Units consist of various administrative units, eg U1 Accounting, U14 Statistics, etc. Table 1 shows the units that accept trainees in the institutions and their daily trainee capacities. The daily total trainee capacity of the institutions is 27 .

According to the curriculum, students must complete three training periods in any unit: 21-day summer training at the end of the 3rd grade and 14-day training in the fall and spring semesters of the 4 th grade.

There are two types of the student group, day (70 students) and night (50 students), subject to the same curriculum. Each student will attend the training only a day in a week, during working hours. Since the course schedules of the night students are out of work hours, any day of the week is suitable for training. However, this is the opposite of the day students because the course timetable of the day students coincides with the working hours of the institutions. It should be ensured that the course days and the training days of the students do not coincide. 


\begin{tabular}{|c|c|c|c|c|c|c|c|c|c|c|c|c|c|c|c|c|c|}
\hline \multirow{6}{*}{ 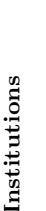 } & & \multicolumn{15}{|c|}{ Units } & \multirow[b]{2}{*}{ U16 } \\
\hline & & U1 & U2 & U3 & U4 & U5 & U6 & U7 & U8 & U9 & U10 & U11 & U12 & U13 & U14 & U15 & \\
\hline & I1 & 3 & 3 & - & - & - & - & - & - & - & - & - & - & - & - & - & - \\
\hline & I2 & - & - & 2 & 3 & - & - & - & - & - & - & - & - & - & - & - & \\
\hline & I3 & - & - & - & - & 2 & 1 & 2 & 1 & 1 & 1 & 2 & 1 & 1 & 1 & - & - \\
\hline & I4 & - & - & - & - & - & - & 1 & - & - & - & - & - & - & - & 1 & 1 \\
\hline
\end{tabular}

- indicates that the relevant unit is not available in the relevant institution.

Table 1: Daily trainee capacities of units in institutions

Considering that the entire capacity is allocated only to the day students, it is clear that the course schedule of these students is off approximately $3(70 / 27=2.59)$ days a week. The courses required by the curriculum must place for the remaining 2 days. The educator has to fit the practical training days of the day group students into 3 days to avoid conflict with the course schedule and also ensure that all students attend the practical training one day a week.

\subsection{The Rotation Strategies}

The rotation strategy currently underway is as follows. The educator assigns trainees to units for the fall and spring periods manually, regardless of the summer training. The trainee may practice at any unit in the fall semester. However, in the spring semester, the trainee could not practice in the same unit of the institution in which trained in the fall semester but may practice in the same unit of different institutions. For example, U7 exists in I3 and I4 institutions (see Table 1). If the trainee is trained in the U7 unit of the I3 institution during the fall semester, the trainee can not practice in the U7 unit of the I3 institution during the spring semester, but there is no obstacle to practice in the U7 unit of the I4 institution. The underlying idea of this approach is that, although the units perform the same function, the internal operation of the units will be different because the institutions have different missions.

The goal of the current rotation strategy is to just provide constraints. This process, which does not require any optimization, is done manually and takes about half a day with the checks. In line with the literature, we aim to maximize the benefits of the rotation plan with optimization, taking into account the preferences of trainees. We have identified eight rotation strategies by combining different sets of rules and different practical training periods. Table 2 summarize rotations strategies. We can explain briefly the rule sets as follows. As emphasized before, a Suitable Course Schedule is required in order not to overlap between course days and training days. Unit Preferences of Trainees states that the unit preferences of the trainee are taken into account. Considering Summer Training indicates that the unit in which summer training is held is taken into account. Different Unit in Each Training Period means that the trainee must be trained in a different unit during each training period. The existence of this rule enables the trainee to increase experience by training in different units as much as possible, and its absence allows the trainee to train in the same unit during all training periods and specialize in a single unit.

In terms of training periods, the default setting is a 3-period: 21 days in summer, 14 days in fall, and 14 days in spring. The fall and spring semesters can be divided into two until the midterm exams and evaluated as 7-day periods. In this case, the 21-14-7-7 setting is 4-period; formed by dividing the spring semester into two and the 21-7-7-7-7 setting is 5-period; formed by dividing the fall and the spring terms into two. There is an inverse relationship between the number of periods and the length of the period. The high number of periods means that the trainee can practice in many units, but this practice will take a relatively short time, which limits the experience they will gain on a unit basis.

We measure the performance of the strategies with three criteria: the level of satisfaction of preferences $(\mathrm{C} 1)$, the number of training units $(\mathrm{C} 2)$, and the number of training days in the 
The Curricular Practical Training Rotation Problem Formulation and the Assessment of Rotation Strategies 123

\begin{tabular}{|c|c|c|c|c|c|c|c|c|c|}
\hline Rules & R1 & R2 & R3 & R4 & R5 & R6 & R3 & R7 & R8 \\
\hline Suitable Course Schedule & + & + & + & + & + & + & + & + & + \\
\hline Unit Preferences of Trainees & & + & + & + & + & + & + & + & + \\
\hline Considering Summer Training & & & + & + & + & + & + & + & + \\
\hline \multirow{2}{*}{\multicolumn{10}{|c|}{$\begin{array}{r}\text { Different Unit in Each Training Period } \\
\text { Training Periods }\end{array}$}} \\
\hline & & & & & & & & & \\
\hline $21-14-14$ & + & + & + & + & & & + & & \\
\hline $21-14-7-7$ & & & & & + & & & + & \\
\hline $21-7-7-7-7$ & & & & & & + & & & + \\
\hline
\end{tabular}

Table 2: Rotation Strategies

trained units (C3). C1 is an indicator of to what extent the rotation plan fulfills students' unit preferences. $\mathrm{C} 2$ is the indicator of how many different units the student received training in total. C3 is an indicator of the student's experience level in training units.

$\mathrm{R} 1$ is the same as the current rotation strategy. Automatically prepares the rotation plan is prepared manually by the educator. Others are alternative strategies to create a more efficient rotation plan. Rotation strategies from $\mathrm{R} 1$ to $\mathrm{R} 4$ are created by gradually adding rules and allowing the rules to measure the impact on performance criteria, regardless of the change in the training period. Rotation strategies from R4 to R6 cover all the rules taken into account, allowing the measurement of the impact of changes in the training period on performance criteria. The R3, R7, and R8 strategies cover other rules, except for the Different Unit in Each Training Period rule, and allow the measurement of the effect of changes in the training period on performance criteria.

The mathematical model used to automate the rotation plan and compare rotation strategies is described in Section 2.2.

\subsection{Mathematical Formulation}

This section introduces the mathematical model in which all rules are active. Depending on the strategy, some rules can be passive. Therefore, changes in the mathematical model according to the strategy are expressed at the end of the section.

\section{Sets and Indices}

$\begin{array}{ll}S P: \text { Practical Training Periods } & p: \text { indices of } S P \\ S: \text { Trainees } & s: \text { indices of } S \\ T: \text { Program type of trainees } & t: \text { indices of } T \\ I: \text { Institutions } & i \text { : indices of } I \\ U: \text { Units } & u \text { : indices of } U \\ I U_{i}: \text { Units in Institutions } i & u \text { : indices of } I U_{i} \\ D: \text { Training Days } & d: \text { indices of } D \\ T_{s}: \text { Units in Institutions } i & \end{array}$




\section{Parameters}

$$
\begin{aligned}
& C_{i u}: \text { Trainee capacity of each unit } u \text { in institution } i \\
& S_{\text {summer }}: \text { The unit where trainees attend summer practice } \\
& S_{s u}: \text { Preference score of trainee } s \text { for unit } u
\end{aligned}
$$

\section{Decision Variables}

$z_{p s i}: 1$ if trainee $s$ is assigned to institution $i$ at period $p, 0$ otherwise

$y_{p s i u}: 1$ if trainee $s$ is assigned to unit $u$ of institution $i$ at period $p, 0$ otherwise

$x_{\text {psiud }}: 1$ if trainee $s$ is assigned to training day $d$ of unit $u$ of institution $i$ at period $p, 0$ otherwise

$q_{p t d}: 1$ if any trainee of program type $t$ is assigned to training day $d$ at period $p, 0$ otherwise $w_{s u}: 1$ if trainee $s$ is assigned to unit $u$

\section{Objective Function}

$$
\begin{aligned}
& \min z_{1}: \sum_{p \in S P} \sum_{t \in T} \sum_{d \in D} q_{p t d} \\
& \max z_{2}: \sum_{p \in S P} \sum_{s \in S} \sum_{i \in I} \sum_{u \in I U_{i}} S_{s u} * y_{p s i u}
\end{aligned}
$$

The model has two objectives. Eq. (1) minimizes the days that trainees of all program types receive training to ensure the Suitable Course Schedule rule. Eq. (2) ensures that trainees are assigned to the units they prefer as much as possible by the Unit Preferences of Trainees rule.

\section{Constraints}

$$
\begin{aligned}
& \sum_{i \in I} z_{p s i}=1 \\
& \forall p \in S P, s \in S \\
& \sum_{u \in I U_{i}} y_{p s i u}=z_{p s i} \\
& \forall p \in S P, s \in S, i \in I \\
& \sum_{d \in D} x_{p s i u d}=y_{p s i u} \\
& \sum_{p \in S P} \sum_{i \in I} y_{p s i u}=0 \\
& \forall p \in S P, s \in S, i \in I, u \in I U_{i} \\
& \sum_{p \in S P} y_{p \operatorname{siu}} \leq 1 \\
& \sum_{s \in S: T_{s}=t} \sum_{i \in I} \sum_{u \in I U_{i}} x_{p s i u d} \leq \sum_{i \in I} \sum_{u \in I U_{i}} C_{i u} * q_{p t d} \\
& \sum_{s \in S} x_{p s i u d} \leq C_{i u} \\
& \sum_{p \in S P} \sum_{i \in I} \sum_{u^{\prime} \in I U_{i}: u^{\prime}=u} y_{p s i u^{\prime}} \leq w_{s u} \\
& w_{s u}=1 \\
& \forall s \in S, u=S_{\text {summer }} \\
& \sum_{u \in U} w_{s u}=1+|S P| \\
& \forall s \in S
\end{aligned}
$$


Eq. (3-5), respectively, ensures that, in each training period, each trainee is assigned to only one institution, assigned to only one unit of the institution to which they are assigned, and receive training for only one day in the unit to which they are assigned. Eq. (6) prevents the trainee from being assigned to the unit where they practice in the summer period, in the fall and spring terms. Eq. (7) ensures that the trainee can receive training at most once in the same unit of the same institution in all training periods. Eq. (8) prevents the number of trainees assigned to each training day for students of each program type in each training period from exceeding the total daily trainee capacity. Eq. (9) limits the maximum number of trainees that can be assigned to a unit by capacity. Eq. (10-12) are constraints that complement each other. Eq. (10) follows the units in which the trainee practised, regardless of the planning period and institution. Eq. (11) ensures that the trainee's unit of training in summer practice is determined. Eq. (12) ensures that each trainee is assigned to a unit in all planning periods (where 1 summer practice and $|S P|$ refers to \# of practical training periods).

\begin{tabular}{cc} 
Strategies & Passive Equations \\
\hline $\mathbf{R} 1$ & $2,6,12$ \\
$\mathbf{R 2}$ & 6,12 \\
$\mathbf{R 3}$ & 12 \\
$\mathbf{R} 4$ & - \\
\hline
\end{tabular}

Table 3: Strategy based passive equations of the mathematical model

We have four main strategies based on the rules (R1, R2, R3, R4). Table 3 shows which equation is passive in which strategy. The variation in the training period is insignificant as it takes place as a parameter in the mathematical model.

\section{Comparison of Rotation Strategies}

In this section, we compare rotation strategies and assess their performance. Two parameters depending on the students are given to the mathematical model. The first is the summer training unit of trainees, and the second is the unit preferences of trainees. To compare rotation strategies and avoid data bias, we have prepared three different data sets according to these parameters: Equal, Random, Stacked. In the equal data set, summer training units and unit preferences are distributed as equally as possible on a unit basis. In the random data set, summer training units and unit preferences are randomly distributed on a unit basis. In the stacked data set, summer training and unit preferences are created in proportion to the trainee capacity of the units. For example, the capacity of U1 is about $11 \%$ of the total capacity. Accordingly, $11 \%$ of students received summer training at U1 and preferred to train at U1.

In simulating the unit preferences, we score only the top three choices of the trainees to give priority to the preferences and to give preferences as much as possible. The first choice is to score 100 points, the second 50 points, the third 10 points, and the other 0 points.

\section{Criteria}

\section{Orientation}

\begin{tabular}{lll}
\hline C1 & The Level of Satisfaction of Preferences & Max \\
C2 & The Number of Training Units & Max \\
C3 & The Number of Training Days in The Trained Units & Max \\
\hline
\end{tabular}

Table 4: Performance criteria 
Table 4 shows the performance criteria. The strategy score is calculated by taking the average of all trainees from the optimal solution of each strategy as explained below.

The level of satisfaction of preferences score is calculated as the sum of the values obtained by dividing by 100 the unit preference score to which the trainee is assigned during each training period. As the summer training unit is out of the control of the educator, it is not taken into account in the calculation of this criterion. The number of training units score indicates how many different units were trained during all training periods including summer training. The number of training days in the trained unit's score is calculated by reference to the longest training period, summer training. When calculating the score of a trainee in this criterion, it is evaluated as 1.0 points if they have trained for 21 days in a unit, 0.66 points if they have been trained for 14 days, 0.33 points if they have been trained for 7 days, and the total score is divided by the total number of training periods including the summer period.

Table 5 shows the scores of each data set of each rotation strategy based on performance criteria. The Average row indicates the average score of each strategy (max-oriented). The Max-Min row displays the consistency of the strategy against changes in the data set (minoriented).

\begin{tabular}{rcccccccc} 
C1 & $\mathbf{R 1}$ & $\mathbf{R 2}$ & $\mathbf{R 3}$ & $\mathbf{R 4}$ & $\mathbf{R 5}$ & $\mathbf{R 6}$ & $\mathbf{R 7}$ & $\mathbf{R 8}$ \\
\hline Equal & 0.18 & 0.80 & 0.77 & 0.76 & 0.63 & 0.53 & 0.66 & 0.56 \\
Random & 0.16 & 0.84 & 0.82 & 0.80 & 0.66 & 0.55 & 0.69 & 0.58 \\
Stacked & 0.23 & 0.69 & 0.67 & 0.66 & 0.56 & 0.48 & 0.59 & 0.52 \\
Average & 0.19 & 0.77 & 0.75 & 0.74 & 0.62 & 0.52 & 0.65 & 0.55 \\
Max-Min & 0.06 & 0.15 & 0.15 & 0.14 & 0.10 & 0.06 & 0.10 & 0.06 \\
& & & & & & & & \\
C2 & $\mathbf{R 1}$ & $\mathbf{R 2}$ & $\mathbf{R 3}$ & $\mathbf{R 4}$ & $\mathbf{R 5}$ & $\mathbf{R 6}$ & $\mathbf{R 7}$ & $\mathbf{R 8}$ \\
\hline Equal & 2.88 & 2.81 & 2.93 & 3.00 & 4.00 & 5.00 & 3.88 & 4.85 \\
Random & 2.89 & 2.80 & 2.92 & 3.00 & 4.00 & 5.00 & 3.88 & 4.85 \\
Stacked & 2.83 & 2.77 & 2.95 & 3.00 & 4.00 & 5.00 & 3.88 & 4.85 \\
Average & 2.87 & 2.79 & 2.93 & 3.00 & 4.00 & 5.00 & 3.88 & 4.85 \\
Max-Min & 0.06 & 0.04 & 0.02 & 0.00 & 0.00 & 0.00 & 0.00 & 0.00 \\
& & & & & & & & \\
C3 & $\mathbf{R 1}$ & $\mathbf{R 2}$ & $\mathbf{R 3}$ & $\mathbf{R 4}$ & $\mathbf{R 5}$ & $\mathbf{R 6}$ & $\mathbf{R 7}$ & $\mathbf{R 8}$ \\
\hline Equal & 0.82 & 0.80 & 0.75 & 0.77 & 0.58 & 0.46 & 0.59 & 0.46 \\
Random & 0.81 & 0.80 & 0.75 & 0.77 & 0.58 & 0.46 & 0.59 & 0.46 \\
Stacked & 0.84 & 0.83 & 0.76 & 0.77 & 0.58 & 0.46 & 0.59 & 0.46 \\
Average & 0.82 & 0.81 & 0.75 & 0.77 & 0.58 & 0.46 & 0.59 & 0.46 \\
Max-Min & 0.02 & 0.03 & 0.01 & 0.00 & 0.00 & 0.00 & 0.00 & 0.00 \\
\hline
\end{tabular}

Table 5: Comparison of Rotation Strategies

The variability in Max-Min values indicates that $\mathrm{C} 1$ is more sensitive to data set change among performance criteria. When considering the sensitivity of data set change strategies, it is shown that R4, R5, R6, R7, R8 in C2 and C3 criteria and R1, R6, R8 in C1 criteria provide consistent results. In terms of $\mathrm{C} 1$, the best score belongs to the $\mathbf{R 2}, \mathbf{R} 3, \mathbf{R} 4$ strategies with tolerance below 5\%. The best score, in the C2, belongs to the R6 strategy. However, since the Different Unit in Each Training Period rule is active in R4, R5, and R6 strategies, considering the training period conditions they are subject to, each has the best score. If we ignore this rule, the best score belongs to the $\mathbf{R} 8$ strategy. When evaluated in terms of C3 criteria, it is seen that training period formation affects the scores and the best score belongs to R1 and R2 strategies. 
Comparison results show that any strategy does not dominate the others in all criteria. Each strategy has an advantage in different criteria. In line with the relative importance of the criteria that reflect the point of view of the decision-maker, the most appropriate strategy can be determined. For this, we adopt the simple weighted method. As a first step, we normalize the strategies' average scores in Table 5 for each criterion. $\bar{x}_{\text {normalized }}=\left(x-x_{\min }\right) /\left(x_{\max }-x_{\min }\right)$ In the second step, each strategy (SWSS) score is obtained by multiplying each criterion's weight by the normalized average score of each strategy. $S W S S_{R x}=\bar{x}_{c 1, R x} w_{c 1}+\bar{x}_{c 2, R x} w_{c 2}+\bar{x}_{c 3, R x} w_{c 3}$ As the third step, the strategy with the highest score is selected.

\begin{tabular}{ccccccccc} 
Average Scores & $\mathbf{R 1}$ & $\mathbf{R 2}$ & $\mathbf{R 3}$ & $\mathbf{R 4}$ & $\mathbf{R 5}$ & $\mathbf{R 6}$ & $\mathbf{R 7}$ & $\mathbf{R 8}$ \\
\hline C1 & 0.19 & 0.77 & 0.75 & 0.74 & 0.62 & 0.52 & 0.65 & 0.55 \\
C2 & 2.87 & 2.79 & 2.93 & 3.00 & 4.00 & 5.00 & 3.88 & 4.85 \\
C3 & 0.82 & 0.81 & 0.75 & 0.77 & 0.58 & 0.46 & 0.59 & 0.46 \\
\hline Normalized Average Scores & $\mathbf{R 1}$ & $\mathbf{R 2}$ & $\mathbf{R 3}$ & $\mathbf{R 4}$ & $\mathbf{R 5}$ & $\mathbf{R 6}$ & $\mathbf{R 7}$ & $\mathbf{R 8}$ \\
C1 & 0.00 & 1.00 & 0.96 & 0.94 & 0.73 & 0.57 & 0.78 & 0.62 \\
C2 & 0.03 & 0.00 & 0.06 & 0.09 & 0.55 & 1.00 & 0.49 & 0.93 \\
C3 & 1.00 & 0.96 & 0.81 & 0.86 & 0.34 & 0.02 & 0.37 & 0.00 \\
\hline
\end{tabular}

Table 6: Average and Normalized Average Scores of Strategies

The strategies are evaluated with 32 different weight profiles, with a total of 1.0 representing all possible perspectives of the decision-maker. Table 7 shows the scores of each strategy and the ideal strategy for each weight profile. In $69 \%$ profile $(22 / 32)$, the R2 strategy, and in $21 \%(10 / 32)$ the R6 strategy is appropriate. The results show that the strategies R2 and R6 outperform the others. Although R2 is dominated by R6 in a small number of weight profiles, $\mathrm{R} 2$ is in the preferred position in most cases. The prominence of $\mathrm{R} 2$ and $\mathrm{R} 6$ among the strategies shows that there are two main judgments in the planning of rotation. The dominant view R2 has a structure that does not choose between concentrating in a unit or having experience in various units, although it takes into account preferences. R6, which has a minority view, adopts that students should have as much experience as possible in a different unit while taking into account preferences.

The strategy scores are very close in some profiles. The last column of Table 7 shows strategies that fall within the $5 \%$ tolerance of the best score and can be considered as an alternative strategy for the relevant profile. The lack of an alternative strategy in any profile indicates that the best strategy is to dominate the others and to gain an absolute advantage. The existence of many alternative strategies indicates that there is no clear difference between the strategies. While R1 is not included as an alternative strategy in any profile, others have been included as an alternative strategy at least once. R4 is an alternative to R2 in 14 profiles and R8 to R6 in 12 profiles. The balanced profile is $30 \%, 40 \%$, and $30 \%$, in which all strategies except R1, score similarly. When the difference of alternative strategies from role models is examined, a variation is observed based on the Different Unit in Each Training Period rule. R4 tends to increase the number of units trained relative to $\mathrm{R} 2$, while $\mathrm{R} 8$ tends to decrease compared to R6.

\section{Conclusion}

This study introduces the curriculum-based automated practical training rotation problem (CPTRPP). CPTRPP is a variation of the staff assignment problem and is similar to the resident scheduling problem. For students to graduate from various programs, they must complete their 


\begin{tabular}{|c|c|c|c|c|c|c|c|c|c|c|c|c|}
\hline \multicolumn{3}{|c|}{ Weights } & \multicolumn{8}{|c|}{ Simple Weighted Scores of Strategy (SWSS) } & \multirow{2}{*}{ Ideal Strategy } & \multirow{2}{*}{ The ideals with $5 \%$ tolerance } \\
\hline C1 & $\mathrm{C2}$ & C3 & R1 & $\mathbf{R 2}$ & R3 & R4 & R5 & R6 & R7 & R8 & & \\
\hline 0.1 & 0.1 & 0.8 & 0.8 & 0.87 & 0.75 & 0.79 & 0.4 & 0.17 & 0.42 & 0.16 & $\mathrm{R} 2$ & - \\
\hline 0.1 & 0.2 & 0.7 & 0.71 & 0.77 & 0.68 & 0.72 & 0.42 & 0.27 & 0.43 & 0.25 & $\mathrm{R} 2$ & - \\
\hline 0.1 & 0.3 & 0.6 & 0.61 & 0.68 & 0.6 & 0.64 & 0.44 & 0.37 & 0.45 & 0.34 & $\mathrm{R} 2$ & $\mathrm{R} 4$ \\
\hline 0.1 & 0.4 & 0.5 & 0.51 & 0.58 & 0.53 & 0.56 & 0.46 & 0.47 & 0.46 & 0.43 & $\mathrm{R} 2$ & $\mathrm{R} 4$ \\
\hline 0.1 & 0.5 & 0.4 & 0.42 & 0.48 & 0.45 & 0.49 & 0.48 & 0.57 & 0.47 & 0.53 & $\mathrm{R} 6$ & $\mathrm{R} 8$ \\
\hline 0.1 & 0.6 & 0.3 & 0.32 & 0.39 & 0.38 & 0.41 & 0.5 & 0.66 & 0.49 & 0.62 & $\mathrm{R} 6$ & $\mathrm{R} 8$ \\
\hline 0.1 & 0.7 & 0.2 & 0.22 & 0.29 & 0.3 & 0.33 & 0.52 & 0.76 & 0.5 & 0.71 & $\mathrm{R} 6$ & $\mathrm{R} 8$ \\
\hline 0.2 & 0.1 & 0.7 & 0.7 & 0.87 & 0.77 & 0.8 & 0.44 & 0.23 & 0.46 & 0.22 & $\mathrm{R} 2$ & - \\
\hline 0.2 & 0.2 & 0.6 & 0.61 & 0.78 & 0.69 & 0.72 & 0.46 & 0.33 & 0.48 & 0.31 & $\mathrm{R} 2$ & - \\
\hline 0.2 & 0.3 & 0.5 & 0.51 & 0.68 & 0.62 & 0.65 & 0.48 & 0.42 & 0.49 & 0.4 & $\mathrm{R} 2$ & R4 \\
\hline 0.2 & 0.4 & 0.4 & 0.41 & 0.58 & 0.54 & 0.57 & 0.5 & 0.52 & 0.5 & 0.5 & $\mathrm{R} 2$ & $\mathrm{R} 3, \mathrm{R} 4$ \\
\hline 0.2 & 0.5 & 0.3 & 0.32 & 0.49 & 0.47 & 0.49 & 0.52 & 0.62 & 0.51 & 0.59 & $\mathrm{R} 6$ & R8 \\
\hline 0.2 & 0.6 & 0.2 & 0.22 & 0.39 & 0.39 & 0.42 & 0.54 & 0.72 & 0.53 & 0.68 & $\mathrm{R} 6$ & $\mathrm{R} 8$ \\
\hline 0.3 & 0.1 & 0.6 & 0.6 & 0.88 & 0.78 & 0.81 & 0.48 & 0.28 & 0.5 & 0.28 & $\mathrm{R} 2$ & - \\
\hline 0.3 & 0.2 & 0.5 & 0.51 & 0.78 & 0.71 & 0.73 & 0.5 & 0.38 & 0.52 & 0.37 & $\mathrm{R} 2$ & $\mathrm{R} 4$ \\
\hline 0.3 & 0.3 & 0.4 & 0.41 & 0.68 & 0.63 & 0.65 & 0.52 & 0.48 & 0.53 & 0.47 & $\mathrm{R} 2$ & $\mathrm{R} 4$ \\
\hline 0.3 & 0.4 & 0.3 & 0.31 & 0.59 & 0.56 & 0.58 & 0.54 & 0.58 & 0.54 & 0.56 & $\mathrm{R} 2$ & $\mathrm{R} 3, \mathrm{R} 4, \mathrm{R} 5, \mathrm{R} 6, \mathrm{R} 7, \mathrm{R} 8$ \\
\hline 0.3 & 0.5 & 0.2 & 0.22 & 0.49 & 0.48 & 0.5 & 0.56 & 0.67 & 0.56 & 0.65 & $\mathrm{R} 6$ & $\mathrm{R} 8$ \\
\hline 0.3 & 0.6 & 0.1 & 0.12 & 0.4 & 0.41 & 0.42 & 0.58 & 0.77 & 0.57 & 0.74 & $\mathrm{R} 6$ & $\mathrm{R} 8$ \\
\hline 0.4 & 0.1 & 0.5 & 0.5 & 0.88 & 0.8 & 0.82 & 0.52 & 0.34 & 0.55 & 0.34 & $\mathrm{R} 2$ & - \\
\hline 0.4 & 0.2 & 0.4 & 0.41 & 0.78 & 0.72 & 0.74 & 0.54 & 0.44 & 0.56 & 0.43 & $\mathrm{R} 2$ & $\mathrm{R} 4$ \\
\hline 0.4 & 0.3 & 0.3 & 0.31 & 0.69 & 0.65 & 0.66 & 0.56 & 0.53 & 0.57 & 0.53 & $\mathrm{R} 2$ & $\mathrm{R} 3, \mathrm{R} 4$ \\
\hline 0.4 & 0.4 & 0.2 & 0.21 & 0.59 & 0.57 & 0.59 & 0.58 & 0.63 & 0.58 & 0.62 & $\mathrm{R} 6$ & $\mathrm{R} 2, \mathrm{R} 4, \mathrm{R} 7, \mathrm{R} 8$ \\
\hline 0.4 & 0.5 & 0.1 & 0.12 & 0.5 & 0.5 & 0.51 & 0.6 & 0.73 & 0.6 & 0.71 & $\mathrm{R} 6$ & $\mathrm{R} 8$ \\
\hline 0.5 & 0.1 & 0.4 & 0.4 & 0.88 & 0.81 & 0.82 & 0.56 & 0.39 & 0.59 & 0.4 & $\mathrm{R} 2$ & - \\
\hline 0.5 & 0.2 & 0.3 & 0.31 & 0.79 & 0.74 & 0.75 & 0.58 & 0.49 & 0.6 & 0.5 & $\mathrm{R} 2$ & $\mathrm{R} 3, \mathrm{R} 4$ \\
\hline 0.5 & 0.3 & 0.2 & 0.21 & 0.69 & 0.66 & 0.67 & 0.6 & 0.59 & 0.61 & 0.59 & $\mathrm{R} 2$ & $\mathrm{R} 3, \mathrm{R} 4$ \\
\hline 0.5 & 0.4 & 0.1 & 0.11 & 0.6 & 0.59 & 0.59 & 0.62 & 0.69 & 0.63 & 0.68 & $\mathrm{R} 6$ & $\mathrm{R} 8$ \\
\hline 0.6 & 0.1 & 0.3 & 0.3 & 0.89 & 0.83 & 0.83 & 0.59 & 0.45 & 0.63 & 0.47 & $\mathrm{R} 2$ & - \\
\hline 0.6 & 0.2 & 0.2 & 0.21 & 0.79 & 0.75 & 0.75 & 0.62 & 0.54 & 0.64 & 0.56 & $\mathrm{R} 2$ & $\mathrm{R} 3, \mathrm{R} 4$ \\
\hline 0.6 & 0.3 & 0.1 & 0.11 & 0.7 & 0.68 & 0.68 & 0.64 & 0.64 & 0.65 & 0.65 & $\mathrm{R} 2$ & $\mathrm{R} 3, \mathrm{R} 4, \mathrm{R} 7, \mathrm{R} 8$ \\
\hline 0.7 & 0.1 & 0.2 & 0.2 & 0.89 & 0.84 & 0.84 & 0.63 & 0.5 & 0.67 & 0.53 & $\mathrm{R} 2$ & R3 \\
\hline
\end{tabular}

Table 7: Simple Weighted Scores of Strategy

theoretical education with practical training. CPTRPP deals with planning which students will study in which unit during which practical training period.

Our CPTRPP is customized according to the needs expressed by Gümüşhane University Faculty of Health Sciences Department of Health Management. In this case, the rotation plan is prepared manually and only the constraints are met. The quality of the rotation plan is not optimal due to human factors and the complexity of the problem, and the preparation and control of the plan also take time. It is a need to quickly prepare a rotation plan of optimal quality that will satisfy all stakeholders for the case.

In line with the needs, we created various rotation strategies consisting of different rule and training period combinations. We evaluated the rotation quality of the strategies with three performance criteria: the level of satisfaction of preferences, the number of training units, and the number of training days in the trained units. To avoid data bias for comparison, we created different data sets and compared the results. We have adopted the exact solution approach with integer mathematical modeling to ensure that the strategies are implemented optimally and to obtain rotation plans quickly. Neither strategy outperformed others in the comparison results. To distinguish between strategies, we have ranked them with a simple weighting method using 32 weight profiles representing different decision-makers. We determined the strategy with the highest score for each profile as the most appropriate strategy and the ones with a $5 \%$ tolerance as alternative strategies. In this way, we identified the dominant and minority strategies among decision-makers and their alternatives.

The dominant opinion restricts the number of practical training periods and supports students to specialize in the units where they study as much as possible and does not force them to practice in a different unit each period. The alternative makes it compulsory to practice in 
a different unit each period. The minority opinion supports the students to gain experience in as many different units as possible by increasing the number of practical training periods and makes it compulsory to practice in a different unit each period. The alternative follows a similar approach but does not require training in a different unit each period.

Thanks to the methodology followed the decision-maker has the opportunity to determine the appropriate strategy and alternative. Different training period settings, rule sets, and performance criteria can be easily adapted to the methodology. In this respect, the study offers a general solution approach to the CPTRPP problem. Considering the width of the application area addressed by the problem, it is an extremely important contribution. It is believed that the study will be a source of inspiration for decision-makers and researchers in different disciplines and that it will be a pioneer in the literature that will enrich with innovative constraints and objectives stemming from different application types.

\section{References}

[1] Pentico, D. W. (2007). Assignment problems: A golden anniversary survey. European Journal of Operational Research, 176(2), 774-793. doi: 10.1016/j.ejor.2005.09.014

[2] Carraresi, P., \& Gallo, G. (1984). A multi-level bottleneck assignment approach to the bus drivers' rostering problem. European Journal of Operational Research, 16(2), 163-173. doi: 10.1016/03772217(84)90071-7

[3] Bianco, L., Bielli, M., Mingozzi, A., Ricciardelli, S., \& Spadoni, M. (1992). A heuristic procedure for the crew rostering problem. European Journal of Operational Research, 58(2), 272-283. doi: 10.1016/0377-2217(92)90213-S

[4] Gardner, J. C., Huefner, R. J., \& Lotfi, V. (1990). A Multiperiod Audit Staff Planning Model Using Multiple Objectives: Development and Evaluation*. Decision Sciences, 21(1), 154-170. doi: 10.1111/j.1540-5915.1990.tb00322.x

[5] Qin, H., Ming, W., Zhang, Z., Xie, Y., \& Lim, A. (2015). A tabu search algorithm for the multi-period inspector scheduling problem. Computers and Operations Research, 59, 78-93. doi: $10.1016 /$ j.cor.2015.01.003

[6] Zamorano, E., \& Stolletz, R. (2017). Branch-and-price approaches for the Multiperiod Technician Routing and Scheduling Problem. European Journal of Operational Research, 257(1), 55-68. doi: 10.1016/j.ejor.2016.06.058

[7] Chen, X., Thomas, B. W., \& Hewitt, M. (2017). Multi-period technician scheduling with experience-based service times and stochastic customers. Computers and Operations Research, 82, 1-14. doi: 10.1016/j.cor.2016.12.026

[8] Franz, L. S., \& Miller, J. L. (1993). Scheduling Medical Residents to Rotations: Solving the Large-Scale Multiperiod Staff Assignment Problem. Operations Research, 41(2), 269-279. doi: 10.1287 /opre.41.2.269

[9] Castaño, F., \& Velasco, N. (2020). Exact and heuristic approaches for the automated design of medical trainees rotation schedules. Omega (United Kingdom), 97, 102107. doi: 10.1016/j.omega.2019.102107

[10] Bard, J. F., Shu, Z., Morrice, D. J., \& Leykum, L. K. (2017). Constructing block schedules for internal medicine residents. IISE Transactions on Healthcare Systems Engineering, 7(1), 1-14. doi: $10.1080 / 19488300.2016 .1255284$

[11] Topaloglu, S. (2006). A multi-objective programming model for scheduling emergency medicine residents. Computers and Industrial Engineering, 51(3), 375-388. doi: 10.1016/j.cie.2006.08.003

[12] Smalley, H. K., \& Keskinocak, P. (2016). Automated medical resident rotation and shift scheduling to ensure quality resident education and patient care. Health Care Management Science, 19(1), 66-88. doi: 10.1007/s10729-014-9289-8

[13] Proano, R. A., \& Agarwal, A. (2018). Scheduling internal medicine resident rotations to ensure fairness and facilitate continuity of care. Health Care Management Science, 21(4), 461-474. doi: 10.1007/s10729-017-9403-9

[14] Turner, J., Kim, K., Mehrotra, S., DaRosa, D. A., Daskin, M. S., \& Rodriguez, H. E. (2013). Using optimization models to demonstrate the need for structural changes in training programs for 
surgical medical residents. Health Care Management Science, 16(3), 217-227. doi: 10.1007/s10729013-9230-6

[15] Rodriguez, H., Turner, J. P., Speicher, P., Daskin, M. S., \& Darosa, D. (2010). A model for evaluating resident education with a focus on continuity of care and educational quality. Journal of Surgical Education, 67(6), 352-358. doi: 10.1016/j.jsurg.2010.09.004

[16] Brunner, J. O., Bard, J. F., \& Kolisch, R. (2011). Midterm scheduling of physicians with flexible shifts using branch and price. IIE Transactions (Institute of Industrial Engineers), 43(2), 84-109. doi: 10.1080/0740817X.2010.504685

[17] Brunner, J. O., \& Edenharter, G. M. (2011). Long term staff scheduling of physicians with different experience levels in hospitals using column generation. Health Care Management Science, 14(2), 189-202. doi: 10.1007/s10729-011-9155-x

[18] Sherali, H. D., Ramahi, M. H., \& Saifee, Q. J. (2002). Hospital resident scheduling problem. Production Planning and Control, 13(2), 220-233. doi: 10.1080/09537280110069667

[19] Fügener, A., Brunner, J. O., \& Podtschaske, A. (2015). Duty and workstation rostering considering preferences and fairness: a case study at a department of anaesthesiology. International Journal of Production Research, 53(24), 7465-7487. doi: 10.1080/00207543.2015.1082667 